\title{
NUMERICAL RANGE FOR CERTAIN CLASSES OF OPERATORS
}

\author{
RICHARD BOULDIN
}

\begin{abstract}
We prove a basic result which relates the structure of the spectrum to the interior of the numerical range. Using this result we derive corollaries concerning compact operators, quasinilpotents, and finite dimensional operators. In particular, we characterize finite dimensional convexoid operators.
\end{abstract}

1. Introduction. In this paper "operator" will mean a bounded linear transformation of the complex Hilbert space $H$ into itself. The thrust of the conclusions that we obtain here is to show that the numerical range of an operator $T$ can be described provided that $T-z I$ has closed range. For a general discussion of numerical range see Chapter 17 of [4].

2. Preliminaries. For an isolated eigenvalue $z$ there are two different notions of eigenspace; the geometric eigenspace is just the kernel of $T-{ }_{z} I$ (which we simply write as $T-z$ ). The algebraic eigenspace associated with $z$ is the range of an idempotent $P$ defined by a contour integral according to the Banach space operational calculus (see pp. 178-181 of [5], for example). If the underlying Hilbert space is $H$ then both $P H$ and $(I-P) H$ are invariant under $T$, and the restriction of $T-z$ to $P H$ (which we denote $T-z(P H)$ is quasi-nilpotent. We shall say that an eigenvalue is a normal eigenvalue if the corresponding geometric eigenspace reduces $T$; if a normal eigenvalue is an isolated eigenvalue and the geometric multiplicity agrees with the algebraic multiplicity then we say that it is a normal-isolated eigenvalue. Clearly an isolated eigenvalue for a normal operator is a normal-isolated eigenvalue.

It will be convenient to denote by $W(T)$ the numerical range of $T$, i.e. $\{\langle T f, f\rangle:\|f\|=1\}$, and the closure of the numerical range is denoted by $W(T)^{-}$. Finally, an operator is convexoid provided that $W(T)^{-}$is the convex hull of the spectrum of $T$, denoted conv $\sigma(T)$. Note that it follows from Theorem 1.24 on p. 16 of [8] that every point of $\operatorname{conv} \sigma(T)$ can be

Received by the editors August 9, 1971 and, in revised form, November 16, 1971. AMS 1969 subject classifications. Primary 4710, 4615; Secondary 4730, 3735.

Key words and phrases. Hilbert space operator, numerical range, eigenvalues, convex set, extreme points, closed range.

(c) American Mathematical Society 1972 
written as a convex linear combination of some four points of $\sigma(T)$. From this and the fact that the continuous image of a compact set is compact, it follows that conv $\sigma(T)$ is compact. Thus conv $\sigma(T)$ is closed.

\section{Main results.}

LEMMA 1. If $z$ is an eigenvalue of $T$ and if $z$ belongs to the topological boundary of $W(T)$, denoted Bdry $W(T)$, then $\operatorname{ker}(T-z)=\operatorname{ker}\left(T^{*}-z\right)$.

Proof. By choosing an appropriate $\theta$ and replacing $T$ with $e^{i \theta}(T-\bar{z})$ we may assume that $z=0$ and $\operatorname{Re} T \geqq 0$. Then for $f \in \operatorname{ker} T$ we have

$$
0=\operatorname{Re}\langle T f, f\rangle=\langle(\operatorname{Re} T) f, f\rangle=\left\|(\operatorname{Re} T)^{1 / 2} f\right\|^{2}
$$

and so $(\operatorname{Re} T) f=0$. It follows that $f \in \operatorname{ker} T^{*}$.

THEOREM 1. For any $z$ in the spectrum of $T$, i.e. $z \in \sigma(T)$, one of the following holds: (1) $z$ belongs to the interior of the numerical range, i.e. $z \in \operatorname{Int} W(T),(2) z$ is a normal-isolated eigenvalue, (3) the range of $T-z$ is not closed.

Proof. Suppose that $z=0$ and assume that both (1) and (3) above fail. Since $T$ is not invertible either $\operatorname{ker} T \neq\{0\}$ or $\operatorname{ker} T^{*} \neq\{0\}$. In either case zero belongs to Bdry $W(T)$ and the lemma implies that $\operatorname{ker} T=\operatorname{ker} T^{*} \neq$ $\{0\}$. Thus $\operatorname{ker} T$ reduces $T$ and we note that $(\operatorname{ker} T)^{\perp}=\left(\operatorname{ker} T^{*}\right)^{\perp}=T H$. Thus $T=0 \oplus T_{1}$ on $\operatorname{ker} T \oplus T H$ and since $T_{1}$ is one-to-one and onto, it is invertible. It follows that $T-z$ is invertible for all nonzero $z$ with $|z|$ sufficiently small. Hence zero is a normal-isolated eigenvalue for $T$.

REMARK 1. The above theorem can also be deduced from Theorem 1 of Crabb in [3] using the theory of ascent and descent. For an account of the different definitions of numerical range in a Banach space see [1].

COROLlaRY 1. If $T$ is compact and if $z \neq 0$ belongs to $\sigma(T) \cap \operatorname{Bdry} W(T)$ then $z$ is a normal-isolated eigenvalue.

Proof. If $T$ is compact and $z \neq 0$ then $T-z$ has closed range.

For a discussion relevant to the next corollary see Problem 170 of [14].

COROLlaRY 2. If $N$ is a nonzero quasi-nilpotent operator such that $N H$ is closed then $0 \in \operatorname{Int} W(N)$.

Proof. Since $0 \in \sigma(N)$, we can apply Theorem 1 and conclude that either (1) or (2) holds. Since $\sigma(N)=\{0\}$, it is clear that the algebraic eigenspace associated with zero is the whole Hilbert space. So if (2) holds then $N$ is the zero operator.

Consult Lemma 5 of [2] for a list of conditions sufficient for an operator to be convexoid. 
THEOREM 2. If $T$ is convexoid and $z$ is an extreme point of $W(T)^{-}$then either $z$ is a normal-isolated eigenvalue or else $(T-z) H$ is not closed.

Proof. It is clear that the extreme points of $W(T)^{-}$are contained in Bdry $W(T)$ and so this follows from Theorem 1.

In [6] Moyls and Marcus obtain conditions which are necessary and sufficient for an operator on a finite dimensional Hilbert space to be convexoid. The next result is somewhat simpler than their theorem.

COROllary 3. An operator $T$ on a finite dimensional Hilbert space $H$ is convexoid if and only if every extreme point of $W(T)$ is a normal-isolated eigenvalue.

Proof. Half of the corollary is immediate from Theorem 2. We assume that every extreme point of $W(T)$ is a normal-isolated eigenvalue and we denote this set of extreme points by $E(T)$. By the HausdorffToeplitz theorem we know that $W(T)$ is a compact convex set and by the Krein-Milman theorem we know that conv $E(T)=W(T)$. Since $E(T) \subset \sigma(T)$, we have $W(T) \subset \operatorname{conv} \sigma(T)$. It is well known that $W(T)^{-\supset \sigma(T)}$ and since $W(T)$ is closed and convex, we have $W(T) \supset$ conv $\sigma(T)$. Thus the corollary is proved.

The next corollary was also noted by Moyls and Marcus.

COROllary 4. If $T$ is a convexoid operator on $H$ and the dimension of $H$ is not greater than four then $T$ is normal.

Proof. By Corollary $3, W(T)$ has at most four extreme points. If $W(T)$ has four extreme points then $T$ is clearly normal. If $W(T)$ has three extreme points then $T$ has a three dimensional reducing subspace such that $T$ restricted to it is normal. Hence $T$ is the direct sum of a normal operator and an operator on a one dimensional subspace and therefore it is normal. If $W(T)$ does not have more than two extreme points then either $W(T)$ is a line segment or it is one point. In the former case $T$ is a linear function of a selfadjoint operator and in the latter case $T$ is a scalar multiple of the identity. Hence $T$ is normal.

REMARK 2. We give an example due to J. P. Williams which shows that the above corollary cannot be extended to the case where $\operatorname{dim} H$ is five. If the invertible convexoid operator $T$ is normal then $T^{-1}$ is normal and it follows that $T^{-1}$ is convexoid. The operator that we construct is invertible and convexoid but its inverse is not convexoid. Let $T_{0}$ be the matrix operator $\left(\begin{array}{ll}0 & 1 \\ 0 & 0\end{array}\right)$ and recall that the numerical range of $T_{0}$ is the closed disc centered at zero with radius $\frac{1}{2}$. Thus the numerical range of $T_{1}=I+T_{0}$ is the closed disc centered at 1 with a radius of $\frac{1}{2}$. Let $\left\{z_{2}, z_{3}, z_{4}\right\}$ be points so placed that they are the vertices of an equilateral triangle which circumscribes the above disc. If $T_{k}=z_{k} I$ for $k=2,3,4$ and if $T=T_{1} \oplus T_{2} \oplus T_{3} \oplus T_{4}$ 
then $T$ is invertible and convexoid but $T^{-1}$ is not convexoid. (The inverse of $T_{1}$ is $I-T_{0}$.)

ACKNOWLEDGEMENT. I am grateful to the referee for simplifying and strengthening the proof of Theorem 1.

\section{REFERENCES}

1. F. F. Bonsall, B. E. Cain and H. Schneider, The numerical range of a continuous mapping of a normed space, Aequationes Math. 2 (1968), no. 1, 86-93. MR 38 \#552.

2. R. H. Bouldin, The numerical range of a product, J. Math. Anal. Appl. 32 (1970), 459-467. MR 42 \#5079a.

3. M. J. Crabb, Some results on the numerical range of an operator, J. London Math. Soc. 2 (1970), 741-745.

4. P. R. Halmos, A Hilbert space problem book, Van Nostrand, Princeton, N.J., 1967. MR 34 \#8178.

5. T. Kato, Perturbation theory for linear operators, Die Grundlehren der Math. Wissenschaften, Band 132, Springer-Verlag, New York, 1966. MR 34 \#3324.

6. B. N. Moyls and M. D. Marcus, Field convexity of a square matrix, Proc. Amer. Math. Soc. 6 (1955), 981-983. MR 17, 820.

7. C. R. Putman, Commutation properties of Hilbert space operators and related topics, Ergebnisse der Mathematik und ihrer Grenzgebiete, Band 36, Springer-Verlag, New York, 1967. MR 36 \#707.

8. F. A. Valentine, Convex sets, McGraw-Hill Series in Higher Math., McGrawHill, New York, 1964. MR 30 \#503.

Department of Mathematics, University of Georgia, Athens, Georgia 30601 